\title{
Sistema de apoio a decisão para seleção de atividades críticas no gerenciamento de projetos com avaliação multicritério
}

\author{
Caroline Maria Guerra de Miranda (UFPE) carolmm@ufpe.br \\ Rodrigo José Pires Ferreira (UFPE) rodrigo@ufpe.br \\ Ana Paula Henriques de Gusmão (UFPE) anagusmao@ufpe.br \\ Adiel Teixeira de Almeida (UFPE) aalmeida@ufpe.br
}

\section{Resumo}

Este trabalho apresenta um sistema de apoio a decisão para o gerenciamento de projetos utilizando metodologia multicritério. $O$ gerenciamento de projetos é um negócio caracterizado por falhas, geralmente ocasionado por incertezas e julgamentos incorretos das urgências do projeto. A maioria dos gerentes não focaliza sobre os aspectos críticos dos projetos. Considerando esses fatores, construiu-se um sistema de apoio à decisão proposto a avaliar, ao mesmo tempo, todos os aspectos críticos, relevantes, do projeto. Isto é, gerenciar as atividades em função de outros aspectos além do tempo, tais como custo, qualidade, segurança, entre outros. O que se quer é selecionar as atividades mais criticas de um projeto levando em consideração os fatores críticos através do método multicritério ELECTRE.

Palavras-chave: PERT/CPM, multicritério, ELECTRE.

\section{Introdução}

Este artigo apresenta um Sistema de Apoio a Decisão (SAD) desenvolvido para suportar o processo de gerenciamento de grandes projetos, atendendo a múltiplos critérios e envolvendo prazos, custos e aspectos relacionados ao desempenho futuro de uma organização.

Neste trabalho, a gestão de projetos ocorre logo após o início da implementação do projeto e está relacionada ao processo de acompanhamento, envolvendo, além do controle, todo o processo de reavaliação e ajustes que implicam o replanejamento do projeto ao longo do processo de implementação.

O gerenciamento de grandes projetos é uma tarefa difícil, considerando-se a complexidade, as incertezas e o grande número de atividades envolvidas. Esse processo pode ser auxiliado por técnicas de planejamento e controle de projetos PERT/CPM ${ }^{1}$, inicialmente desenvolvidas por engenheiros que trabalhavam com projetos complexos de defesa e construção civil (SLACK et al, 1995).

A técnica PERT/CPM, largamente utilizada para esse tipo de problema, determina o caminho crítico e, consequentemente, as atividades críticas com base nas folgas visualizadas pela rede. Com isso, verifica-se que existe a preocupação com o critério tempo.

Todavia, Greek \& Pullin (1999) destacam que a maioria dos gerentes não focaliza os aspectos críticos dos projetos. Segundo esses autores, o gerenciamento de projetos é um negócio caracterizado por falhas e essas falhas são ocasionadas por duas razões: incertezas de ordem técnica e julgamentos incorretos das urgências do projeto. As falhas não estão diretamente ligadas ao abandono, isso pode até acontecer, mas, por exemplo, estão significativamente relacionadas ao tempo ou ao orçamento.

Considerando esses fatores, o processo de gerenciamento de projetos pode ser suportado por

${ }^{1}$ PERT-Program Evaluation and Review Technique; CPM-Critical Path Method (PREMACHANDRA, 2001) 
métodos de apoio multicritério à decisão, envolvendo o estudo dos aspectos críticos do projeto, isto é, planejar, programar e gerenciar as atividades em função de outros aspectos além do tempo, tais como priorizar atividades de baixa segurança, alto custo, dentre outros aspectos que sejam relevantes para o gerente.

Serão discutidos, nos próximos itens, os conceitos básicos sobre o planejamento e gestão de empreendimentos de forma a caracterizar o problema de gerenciamento de projetos como um problema de apoio a decisão multicritério. Será abordada, então, a metodologia multicritério utilizada e, a seguir, o sistema que foi construído. Por fim serão apresentadas as conclusões desse trabalho.

\section{Analise do problema}

Este item apresenta uma visão geral do problema de gerenciamento de projetos e uma nova forma de tratar esse problema, utilizando a abordagem PERT/CPM associada aos métodos multicritério de apoio à decisão.

Na visão PERT/CPM, um projeto pode ser visualizado como um conjunto de operações conduzidas numa certa seqüência para se atingirem dados objetivos. Identificadas as atividades, elas podem ser representadas e ordenadas em um Digrama de Rede.

A técnica PERT/CPM pode ser utilizada em conjunto com uma metodologia de apoio multicritério à decisão, incorporando os aspectos críticos dos projetos e permitindo tratar o problema de forma mais realista. Nesse caso, as atividades críticas do projeto são determinadas de modo que o gerente possa concentrar seus esforços no controle e acompanhamento das atividades com maior chance de apresentar problemas ao longo da execução.

Para isso, são determinadas as atividades críticas com relação ao tempo (aplicação da técnica PERT/CPM - baseada no cálculo das folgas) e, em seguida, inserem-se os novos critérios alocados ao projeto. As atividades críticas são determinadas mediante a aplicação do método de apoio multicritério à decisão, que considera mais de um critério simultaneamente.

Para a aplicação dos métodos multicritério, podem ser considerados os critérios: custo, mobilização de recursos, duração, folga, variância (quando se utiliza a estimativa de tempo probabilística), dentre outros relevantes para o gerente (MIRANDA \& ALMEIDA, 2002).

Construídas as redes PERT/CPM e definidos os aspectos críticos do projeto (novos critérios), o método de apoio à decisão multicritério pode então ser aplicado em dois momentos distintos, chamados de Primeiro e Segundo Procedimento.

O Primeiro Procedimento tem o objetivo de dar uma visão geral no início do processo de gestão. Nesse caso, um pouco antes de se iniciar a execução do empreendimento, o processo de gestão pode ser aplicado em duas situações diferentes: quando o caminho crítico tem folga igual a zero e quando a folga é diferente de zero.

Quando o caminho crítico do projeto tem folga igual a zero, as atividades desse caminho são consideradas imediatamente críticas, independentemente da aplicação do método multicritério, sendo então retiradas do processo. Na outra situação, todas as atividades são consideradas no problema.

A aplicação do processo de gestão consiste em levantar as avaliações das atividades para todos os novos critérios e também para o critério tempo. Aplica-se o método multicritério e selecionam-se as atividades críticas (GOMES et al, 2002; ROY, 1996; KEENEY \& RAIFFA, 1976; VINCKE, 1996). 
Esse processo de gerenciamento de projetos usando multicritério pode ser visualizado pelo esquema a seguir (Figura 1).

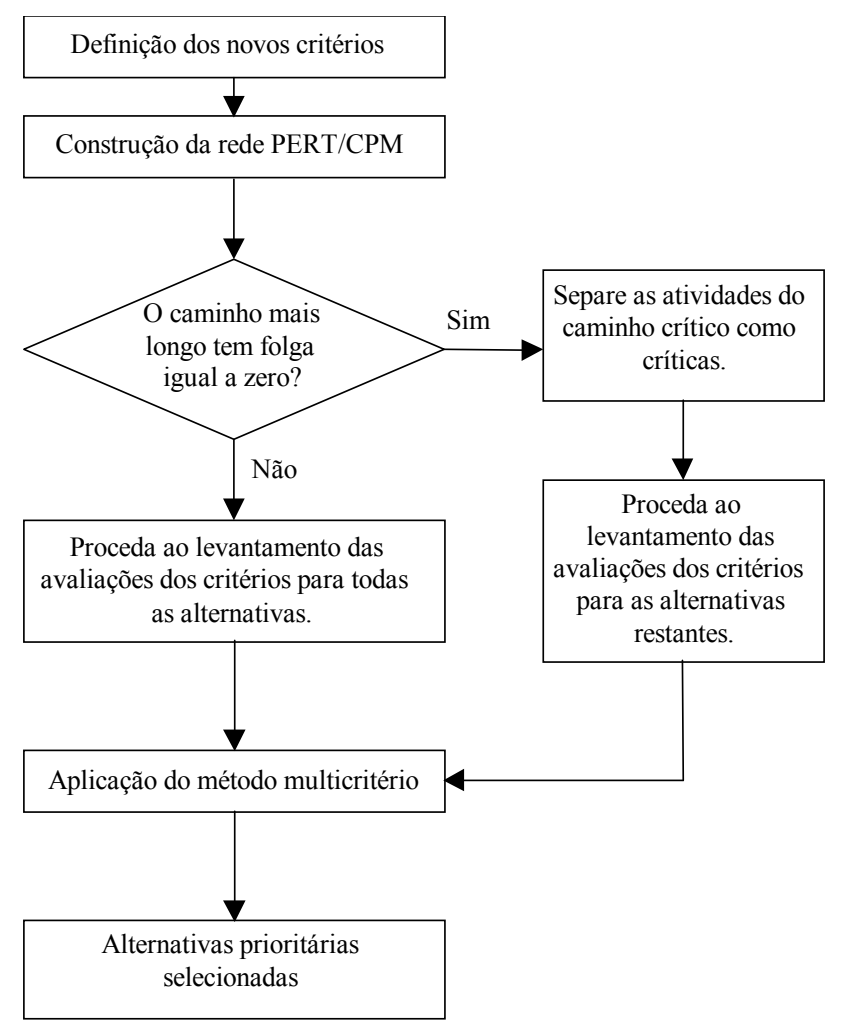

Figura 1 - Processo de Gerenciamento de Projetos.

O segundo procedimento deve ser utilizado periodicamente, conforme seja o momento de estudo (por exemplo, mensal, anual, qualquer). Isso deve ser feito para selecionar as atividades críticas de um período específico e também porque devem ser consideradas as mudanças (replanejamento) ocorridas durante a execução do empreendimento.

Identificadas as atividades do período, são levantados os dados referentes a essas atividades. Cabe lembrar que, devido às incertezas do planejamento, pode haver mudanças no cronograma atual. Nesse caso, algumas atividades que possuíam folga maior que zero, nesse momento, podem apresentar folga zero ou vice-versa. Em outra ocasião, pode ser necessário um replanejamento das atividades devido à inserção de novas informações ou restrições ao processo de execução das atividades.

Estabelecidas as atividades e suas respectivas informações, aplica-se o método de apoio multicritério à decisão. Nesse caso, pode ser necessário fazer ajustes no modelo do processo de gestão, como mudanças em alguns parâmetros. Como resultado, obtêm-se as atividades críticas do período. Essas atividades selecionadas merecem atenção especial.

Encontrado o resultado, o processo de gestão é reaplicado para o período de estudo a seguir, aguardando uma data próxima à execução (por exemplo, se o período de estudo é mensal, o estudo do próximo período pode ser feito na última semana do período atual). Isso é feito até o último período do projeto em estudo.

$\mathrm{Na}$ construção do sistema de apoio a decisão foi considerado o método multicritério ELECTRE 1. A escolha desse método se deve ao enquadramento da problemática - seleção de um subconjunto de ações - e pela "facilidade" por parte do tomador de decisão (gerente do empreendimento) em trabalhar com os parâmetros do modelo. 


\section{Método Multicritério: ELECTRE}

Os métodos ELECTRE (Elimination and Choice Translating Algorithm) fundamentam-se na construção de uma relação de sobreclassificação que incorpora as preferências estabelecidas pelo decisor diante dos problemas e das alternativas disponíveis. A relação de sobreclassificação $\mathrm{S}$ é uma relação binária definida em $\mathrm{A}$ tal que aSb, se a é pelo menos tão boa quanto b. Essa relação não exige a transitividade (ROY, 1974).

Segundo Roy (1996), estes métodos baseiam no estudo de sobreclassificação em uma lógica não compensatória (razão de substituição), com poder de veto usando as noções de concordância e de discordância. As relações de sobreclassificação são construídas de tal forma que uma alternativa é tão boa quanto a outra, nas seguintes condições: Uma maioria suficiente de critérios, considerando as suas importâncias, apóia esta proposição (princípio da concordância) e a oposição da minoria não é considerada forte o suficiente para discordar desta proposição. Estes métodos são caracterizados por duas etapas. Na primeira são construídas as relações de Sobreclassificação, que representam as preferências estabelecidas pelo decisor. Na segunda, estas relações são exploradas a fim de ajudar o decisor a resolver o seu problema.

A família ELECTRE é composta pelos métodos ELECTRE I, II, III, IV, IS e TRI. Tais métodos são também denominados métodos de subordinação (termo em inglês: outranking). Os métodos da família ELECTRE revelam grande potencial de aplicação, devido à flexibilidade ao enquadramento das problemáticas. O ELECTRE I é destinado a problemáticas de seleção (P. $\alpha$ ), o ELECTRE II, III e IV estão enquadrados em problemáticas

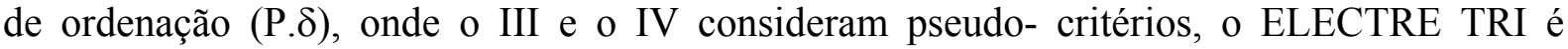
destinado a problemáticas de classificação (P. $\beta$ ) com pseudo- critérios, e por fim o ELECTRE IS está enquadrado em problemáticas de seleção (P. $\alpha$ ) com pseudo- critérios.

Para construção do SAD foi considerado o método ELECTRE I, que é um método que busca eliminar alternativas superadas de acordo com um conjunto de pesos atribuídos pelo decisor a cada objetivo do problema. O método foi construído para problemas de escolha, ou seleção

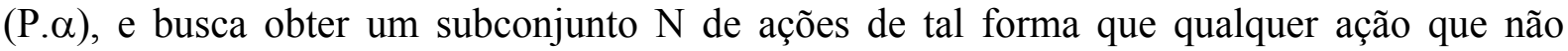
estiver em $\mathrm{N}$ é sobreclassificada por pelo menos uma ação de N. Esse conjunto $\mathrm{N}$ de ações é o conjunto que contém as ações mais satisfatórias à luz dos critérios estabelecidos. (VINCKE, 1992).

Segundo Olson (1996), o ELECTRE I busca reduzir o conjunto de ações (alternativas) A para o menor possível, por meio de índices de concordância e discordância que medem a vantagem e desvantagem relativa par a par entre as alternativas.

A cada critério é atribuído um peso pj que cresce em função da importância relativa do critério e, em seguida, as relações de sobreclassificação são obtidas par a par entre alternativas por meio dos índices de concordância $\mathrm{C}(\mathrm{a}, \mathrm{b})$ e discordância $\mathrm{D}(\mathrm{a}, \mathrm{b})$ e dos seus respectivos limiares de concordância $\mathrm{p}$ e discordância $\mathrm{q}$.

Passada a etapa de construção do modelo, segue-se a etapa de investigação, para encontrar o Kernel. Uma das formas de encontrar o Kernel é fazer uma análise mais refinada das ações do Kernel, ou seja, uma análise de sensibilidade, mediante variações nos pesos dos critérios e nos limiares de concordância e discordância do modelo ( $\mathrm{pj}, \mathrm{p}$ e q ) para verificar sua robustez (VINCKE, 1992; ROY, 1996). 


\section{SAD para seleção de atividades críticas no gerenciamento de Projetos}

Um Sistema de Apoio à Decisão (SAD), é um sistema de informação utilizado para dar suporte a um tomador de decisão em qualquer nível, face a problemas semi-estruturados e não estruturados (DAVIS, 1985; SHIM et al, 2002).

As principais razões que justificam a construção de um SAD são: a complexidade do processo decisório, a relação interativa existente entre o SAD e o usuário, a atenção do decisor voltada para o problema e não para os métodos de resolução, fornecimento rápido das respostas e a possibilidade da geração de cenários, através dos quais o usuário poderá analisar as diversas alternativas de solução do problema.

Nesse contexto, com intuito de auxiliar o processo de gerenciamento de grandes projetos buscando a eficácia da tomada de decisão e, conseqüentemente, da organização foi desenvolvido um Sistema de Apoio à Decisão. Esse sistema foi construído utilizando a linguagem Visual Basic for Applications integrada com uma ferramenta computacional muito utilizada no gerenciamento de projetos, o Microsoft Project 2000.

Para que o SAD auxilie no processo de tomada de decisão é preciso ser composto de uma base de dados - que auxilia o sistema -, uma base de modelos - que provê a capacidade de análise - e o diálogo - que provê a interação entre o usuário e o sistema. Esta é a arquitetura de um SAD.(BIDGOLI,1989; SPRAGUE, 1989). A relação entre esses elementos pode ser visualizada na figura a seguir (Figura 2).

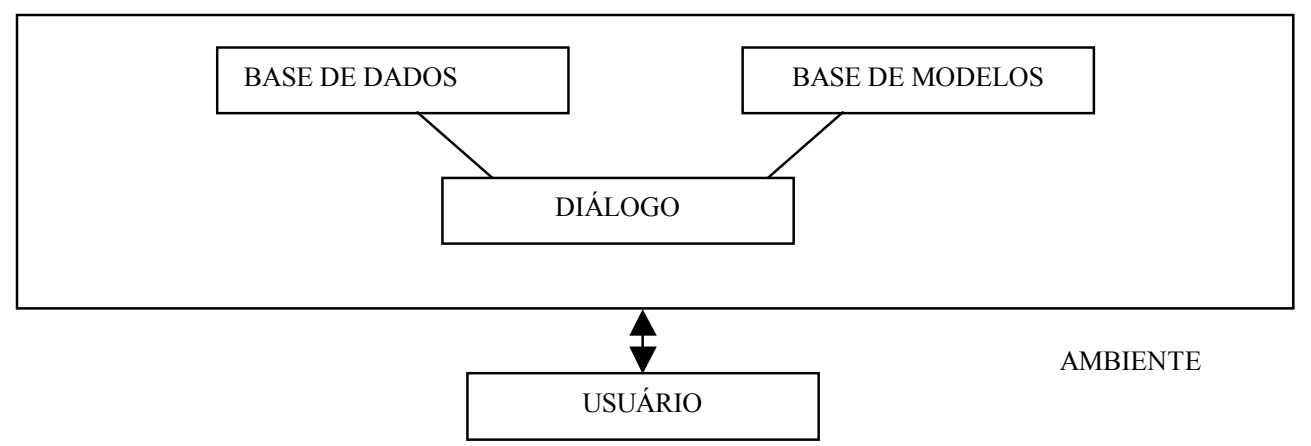

Figura 2 - Arquitetura de um SAD

O elemento base de dados é responsável pelo armazenamento, em um local físico, de dados consistentes e de valor significante para toda a organização. A base de dados do SAD desenvolvido é composta pelos dados provenientes dos projetos em análise, as avaliações das atividades em relação aos critérios considerados e os dados dos parâmetros do modelo, apresentando as preferências do tomador de decisão.

O elemento base de modelos é construído através de uma série de elementos e suas relações (GRAY, 1994). A base de modelos do SAD desenvolvido é composta pelo modelo de decisão multicritério ELECTRE I, apresentado no item anterior. Esta base também permite a análise de cenários.

O diálogo é definido como uma combinação de software, hardware e pessoas, que permite uma interação entre o usuário e o SAD. Trata-se do componente mais importante para o usuário, pois para ele o diálogo é a forma como o sistema opera e funciona. O usuário busca a simplicidade e a funcionalidade da interface, assim, o sistema foi montado a partir do Microsoft Project 2000, considerando que o usuário já está bastante familiarizado com software. As telas do diálogo e o processo de interação com o usuário são apresentados a 
seguir.

Este Sistema de Apoio a Decisão caracteriza-se por uma flexibilidade de operações, com o foco no diálogo direto, que permite o próprio decisor operar o SAD. Há, no entanto, a possibilidade de uma terceira pessoa operar, gerar relatórios e interpretá-los para o decisor, ocorrendo assim um diálogo indireto.

O SAD apóia facilmente aos dois procedimentos do processo de gestão de projetos apresentados no item 2. A execução do aplicativo permite indicar que atividades farão parte do processo de gestão. Para isso é necessário que o decisor faça a avaliação (qualitativa ou quantitativa) de alguns das atividades em relação a alguns critérios considerados.

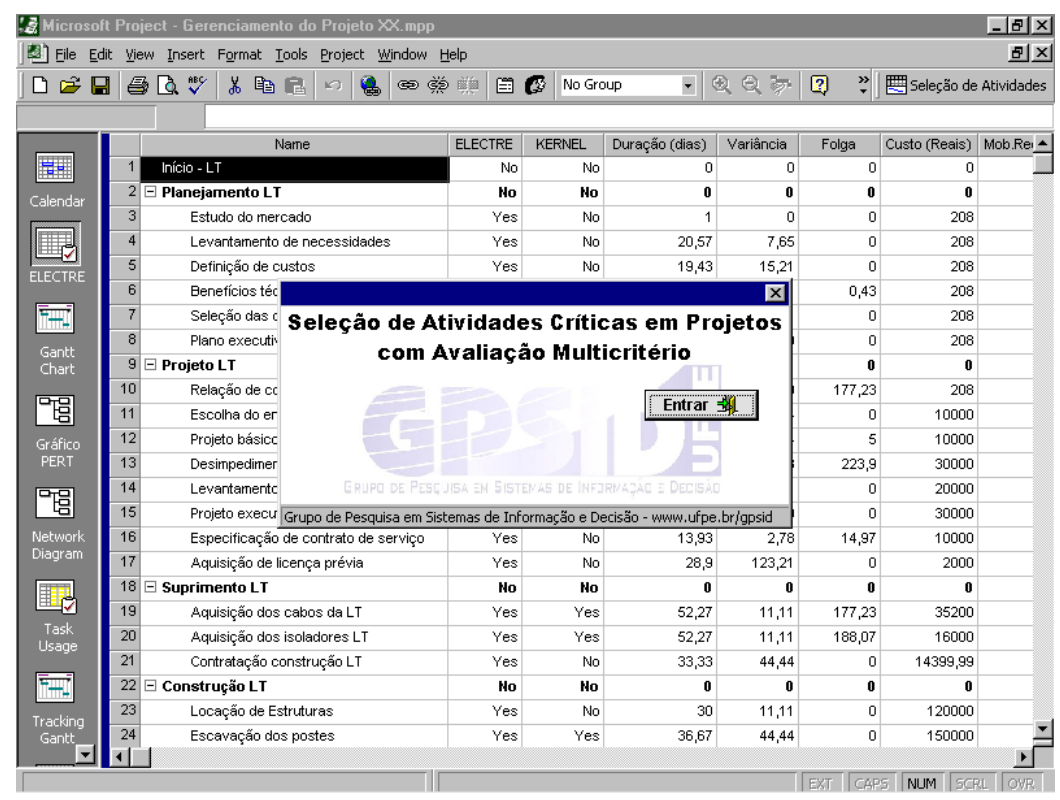

Figura 3 - Tela inicial do Sistema de Apoio à Decisão

A tela principal de interação com o usuário é apresentada abaixo (Figura 4). Essa tela exibe um formulário de entrada de dados e saída dos resultados onde o usuário entrará com os parâmetros nos campos indicados, dando início ao cálculo da seleção das atividades através do método multicritério ELECTRE. Nesse momento o usuário poderá visualizar o resultado diretamente no formulário ou numa coluna de uma planilha do Microsoft Project.

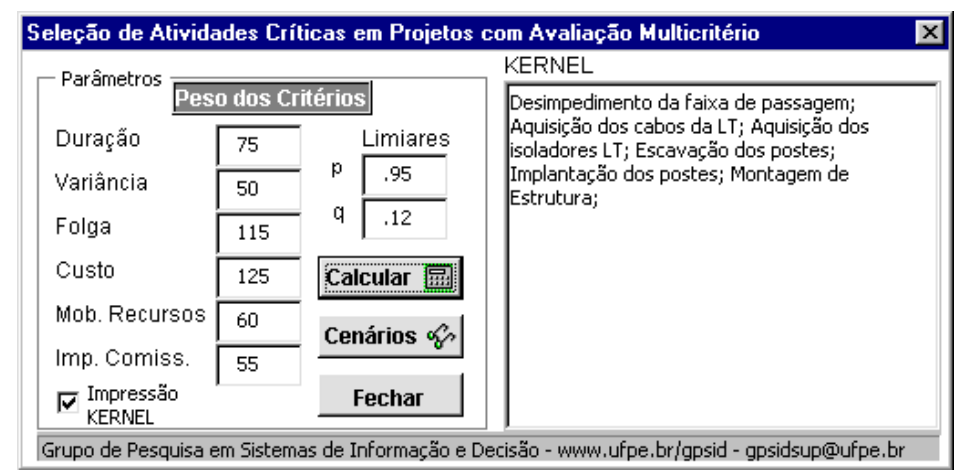

Figura 4 - Formulário de entrada de dados e saída dos resultados

Para uma análise mais detalhada do resultado, o sistema possibilita a geração de cenários, através dos quais os usuários poderão analisar as alterações ocorridas nas variáveis de saída 
em função de modificações dos valores dos parâmetros de entrada. Isto é, poderá ser analisado que atividades permanecem ou não no Kernel quando se faz alterações nas variáveis de entrada do modelo.

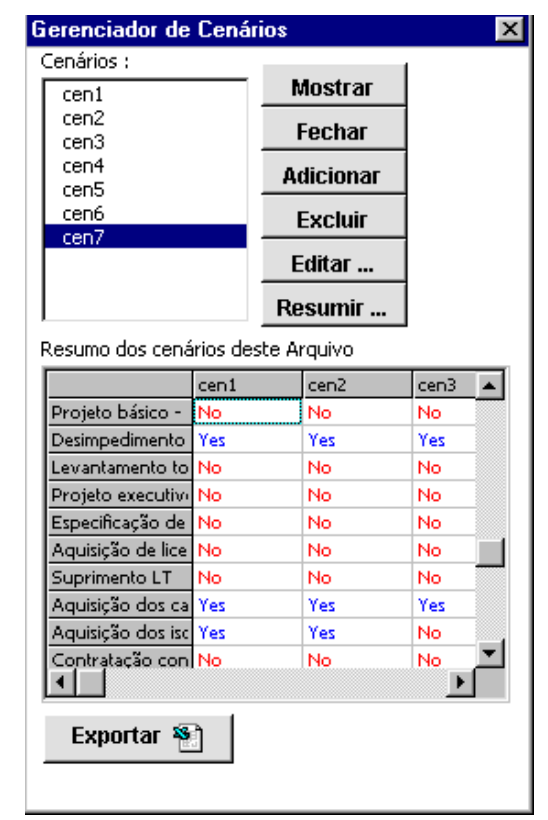

Figura 5 - Gerenciador de Cenários

No formulário da Figura 5, o usuário pode adicionar, excluir, editar e visualizar os resultados de um cenário no formulário inicial. A opção resumir do sistema apresenta os parâmetros de todos os cenários acompanhados de seus respectivos resultados numa matriz. O software possibilita ainda a exportação do resumo para o Microsoft Excel para elaboração de futuros relatórios.

Como resultado desse processo de seleção através do método ELECTRE, obtêm-se um conjunto de atividades que foram consideradas críticas mediante critérios como custo, duração, segurança, além da folga. Observa-se que é necessário um treinamento prévio para os usuários em relação à metodologia multicritério empregada.

\section{Conclusões}

O gerenciamento de grandes projetos, tema que ainda merece grandes discussões, é tratado como um problema de decisão multicritério. A técnica PERT/CPM, muito utilizada para planejar e coordenar projetos, aliada ao método multicritério compõem o processo de gerenciamento de projetos utilizado neste trabalho. Nesse processo, as atividades críticas são avaliadas considerando-se, ao mesmo tempo, todos os aspectos críticos do projeto. Para permitir uma aplicação eficaz desse processo foi construído um sistema de apoio a decisão. Esse sistema complementa as tarefas executadas pelo software Microsoft Project, permitindo maior facilidade em sua aplicação.

O decisor precisa analisar os aspectos críticos do problema e estabelecer as relações entre eles. Para tratar esses aspectos, foi considerado um modelo multicritério com método ELECTRE I, que se mostrou bastante adequado ao problema. O SAD desenvolvido possui alta interatividade e flexibilidade com o usuário, características fundamentais em sistemas desse tipo. Permite ainda a análise de diferentes cenários, o que torna sua aplicação mais refinada. 
Como trabalhos futuros pretende-se avaliar o uso de outros modelos multicritério que trabalhem com problemáticas de seleção ou, em outros casos, avaliar o uso de outra problemática.

\section{Referências}

SPRAGUE JR, R.H.\& WATSON, H.J. (1989) - Decision Support Systems - Putting Theory into Practice. Prentice-Hall, Inc.

BIDGOLI, H. (1989) - Decision Support Systems - Principle and Practice. West Publishing Company.

DAVIS, C.B. \& OLSON M.H. (1985) - Management Information Systems: Conceptual Foundations, Structure and Development. McGraw-Hill.

GOMES, L.F.A.; GOMES, C.F.S. \& ALMEIDA, A.T. (2002) - Tomada de Decisão Gerencial: O Enfoque Multicritério. Rio de Janeiro. Ed. Atlas. Vol.1.

GREEK, D. \& PULLIN, J. (1999) - Overrun, overspent, overlooked. Professional Engineering. Bury St. Edmunds. Vol. 12, n. 3, pp. 27-28.

KEENEY, R. \& RAIFFA, H. (1976) - Decisions with Multiple Objectives - Preferences and Value Trade-offs. John Wiley \& Sons.

OLSON, D.L. (1996) - Decision aids for eletion problems. Springer.

PREMACHANDRA, I. M. (2001) - An approximation of the activity duration distribution in PERT. Computers \& Operations Research. New York. Vol. 28, pg 443-452.

ROY, B. (1996) - Multicriteria Methodology Goes Decision Aiding. Kluwer Academic Publishers.

ROY, B. (1974) - Critères Multiples et Modélisation des Préférences: l'apport des relations de surclassement" Revue d'Economie Politique. APUD Vincke (1992).

SHIM, J.P.; WARKENTIN, M.; COURTNEY, J. F.; POWER, D. J. (2002) - Past, present, and future of decision support technology. In: Decision Support Systems. Vol. 33, pp 111-126.

SLACK, N.; CHAMBERS, S.; HARLAND, C.; HARRISON, A. \& JOHNSON R. (1995) - Administração da Produção. Ed. Atlas.

VINCKE, P. (1992) - Multicriteria Decision-Aid. John Wiley \& Sons Ltd. ISBN: 0-471-93184-5.

\section{Agradecimentos}

Este trabalho foi apoiado parcialmente pelo Conselho Nacional de Desenvolvimento Científico e Tecnológico- CNPq. 